\title{
Quantum chaos and QCD at finite chemical potential
}

\author{
H. Markum ${ }^{\mathrm{a}}$, R. Pullirsch ${ }^{\mathrm{a} *}$, K. Rabitsch ${ }^{\mathrm{a}}$, and T. Wettig ${ }^{\mathrm{b}}$

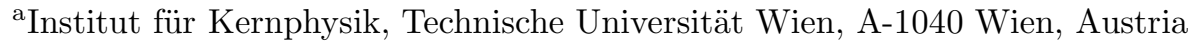 \\ bInstitut für Theoretische Physik, Technische Universität München, D-85747 Garching, Germany
}

We investigate the distribution of the spacings of adjacent eigenvalues of the lattice Dirac operator. At zero chemical potential $\mu$, the nearest-neighbor spacing distribution $P(s)$ follows the Wigner surmise of random matrix theory both in the confinement and in the deconfinement phase. This is indicative of quantum chaos. At nonzero chemical potential, the eigenvalues of the Dirac operator become complex. We discuss how $P(s)$ can be defined in the complex plane. Numerical results from an $\mathrm{SU}(3)$ simulation with staggered fermions are compared with predictions from non-hermitian random matrix theory, and agreement with the Ginibre ensemble is found for $\mu \approx 0.7$.

\section{Introduction}

The spectrum of the QCD Dirac operator, both in the continuum and on the lattice, has several universal properties. By "universal" one means "independent of the details of the dynamics", e.g., independent of the precise values of the simulation parameters on the lattice. Such universal features can be described by random matrix theory (RMT). In this contribution, we are concerned with the eigenvalue fluctuations in the bulk of the spectrum on the scale of the local mean level spacing, measured by the distribution $P(s)$ of the spacings $s$ of adjacent eigenvalues. We will consider gauge group $\mathrm{SU}(3)$ and staggered fermions which are related to the chiral unitary ensemble of RMT. At zero chemical potential $\mu$, all Dirac eigenvalues are purely imaginary, and it has been shown in lattice simulations that $P(s)$ agrees with the Wigner surmise of RMT,

$P_{\mathrm{W}}(s)=\frac{32}{\pi^{2}} s^{2} e^{-\frac{4}{\pi} s^{2}}$,

both in the confinement and in the deconfinement phase [1]. This result implies that the Dirac eigenvalues are strongly correlated, and is indicative of quantum chaos, according to the conjecture by Bohigas, Giannoni, and Schmit 2]. In contrast, quantum systems whose classical analogs are integrable obey a Poisson distribution, $P_{\mathrm{P}}(s)=e^{-s}$.

${ }^{*}$ Poster presented by R. Pullirsch
Here, we focus on nonzero chemical potential. For $\mu \neq 0$, the lattice Dirac matrix generalizes to $M_{x, y}(U, \mu)=\frac{1}{2 a} \sum_{\nu=\hat{x}, \hat{y}, \hat{z}}\left[U_{\nu}(x) \eta_{\nu}(x) \delta_{y, x+\nu}-\right.$ h.c. $]$
$+\frac{1}{2 a}\left[U_{\hat{t}}(x) \eta_{\hat{t}}(x) e^{\mu} \delta_{y, x+\hat{t}}-U_{\hat{t}}^{\dagger}(y) \eta_{\hat{t}}(y) e^{-\mu} \delta_{y, x-\hat{t}}\right]$, with the Kawamoto-Smit phases $\eta$. The eigenvalues of this matrix are complex. In this case, $P(s)$ represents the spacing distribution of nearest neighbors in the complex plane. For each eigenvalue $z_{0}$ one has to find the eigenvalue $z_{1}$ for which $s=\left|z_{1}-z_{0}\right|$ is smallest, with a subsequent average over $z_{0}$. This definition assumes that the spectral density is constant over a bounded region in the complex plane (and zero outside). Generally, this is not the case so that an unfolding procedure must be applied, see Sec. 2. However, the spectral density of the so-called Ginibre ensemble of RMT, where real and imaginary parts of the eigenvalues have the same average size, is constant inside a circle and zero outside, respectively [3]. In this case, $P(s)$ is given by [4]

$P_{\mathrm{G}}(s)=c p(c s)$,

with

$p(s)=2 s \lim _{N \rightarrow \infty}\left[\prod_{n=1}^{N-1} e_{n}\left(s^{2}\right) e^{-s^{2}}\right] \sum_{n=1}^{N-1} \frac{s^{2 n}}{n ! e_{n}\left(s^{2}\right)}$,
$e_{n}(x)=\sum^{n} x^{m} / m !$, and $c=\int_{0}^{\infty} d s s p(s)=$ $1.1429 \ldots$ 

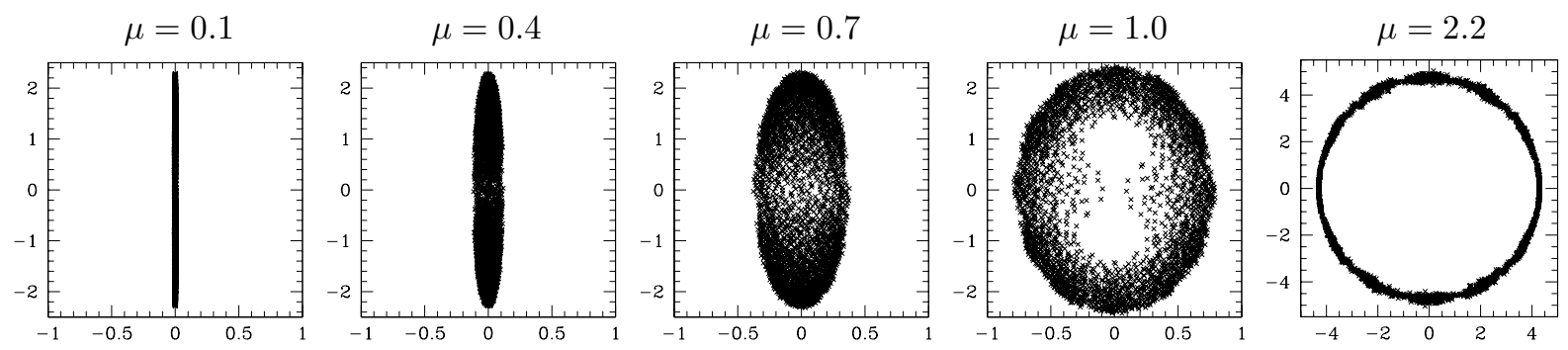

Figure 1. Complex eigenvalues of the Dirac operator at various values of $\mu$ for a typical equilibrium configuration of QCD with staggered quarks without chemical potential (horizontal axes = real parts, vertical axes $=$ imaginary parts, in units of $1 / a$ ).

In contrast, the Poisson distribution in the complex plane, representing uncorrelated eigenvalues, becomes

$P_{\overline{\mathrm{P}}}(s)=\frac{\pi}{2} s e^{-\frac{\pi}{4} s^{2}}$.

In the following, we will study the Dirac spectrum on the lattice at various values of $\mu \neq 0$ and compare the resulting $P(s)$ with Eqs. (2) and (3).

\section{Analysis of complex spectra}

While the quenched approximation at $\mu \neq 0$ is unphysical [5], there is currently no feasible solution to the problem of a complex weight function. Therefore, we generated the gauge field configurations at $\mu=0$ and added the chemical potential to the Dirac matrix afterwards. The simulations were done with gauge group $\mathrm{SU}(3)$ on a $6^{3} \times 4$ lattice using $\beta=5.2$ and $N_{f}=3$ flavors of staggered fermions of mass $m a=0.1$. For each parameter set, we sampled 50 independent configurations.

The eigenvalue spectrum is shown in Fig. 1 for five different values of $\mu$. The size of the real parts of the eigenvalues grows with $\mu$ as expected. While the spectrum is confined to a bounded region in the complex plane, the spectral density is certainly not constant. Therefore, the spectrum has to be unfolded. In one dimension, unfolding is a local rescaling of the eigenvalue density such that the density on the unfolded scale is equal to unity. To the best of our knowledge, unfolding in the entire complex plane has not been considered yet (in Ref. [6], unfolding was done in strips perpendicular to the real axis). We proceed as follows. The spectral density has an average and a fluctuating part, $\rho(x, y)=\rho_{\mathrm{av}}(x, y)+\rho_{\mathrm{fl}}(x, y)$. Unfolding means to find a map $z^{\prime}=x^{\prime}+i y^{\prime}=u(x, y)+i v(x, y)$ such that $\rho_{\text {av }}\left(x^{\prime}, y^{\prime}\right) \equiv 1$. Since the probability has to be invariant, $\rho_{\text {av }}\left(x^{\prime}, y^{\prime}\right) d x^{\prime} d y^{\prime}=d x^{\prime} d y^{\prime}=$ $\rho_{\text {av }}(x, y) d x d y$, we find that $\rho_{\text {av }}(x, y)$ is the Jacobian of the transformation from $(x, y)$ to $\left(x^{\prime}, y^{\prime}\right)$, $\rho_{\text {av }}(x, y)=\left|\partial_{x} u \partial_{y} v-\partial_{y} u \partial_{x} v\right|$. Choosing $y^{\prime}=$ $v(x, y)=y$ yields $\rho_{\mathrm{av}}(x, y)=\left|\partial_{x} u\right|$ and, thus, $x^{\prime}=u(x, y)=\int_{-\infty}^{x} d t \rho_{\mathrm{av}}(t, y)$. Essentially, this is a one-dimensional unfolding in strips parallel to the real axis. For a fixed bin in $y, \rho_{\text {av }}(x, y)$ is determined by fitting $\rho(x, y)$ to a low-order polynomial. $P(s)$ is then constructed from the constant unfolded density as explained in Sec. 11, normalized such that $\int_{0}^{\infty} d s s P(s)=1$.

Several remarks are in order. (i) We have checked spectral ergodicity. If only parts of the spectral support are considered, the results for $P(s)$ do not change. (ii) If the spectral density has "holes" (see Fig. 11 for $\mu=1.0$ and 2.2), we split the spectral support into several convex pieces and unfold them separately. This is justified by spectral ergodicity. (iii) Unfolding each spectrum separately and ensemble unfolding yield the same results for $P(s)$. (iv) The results for $P(s)$ are stable under variations of the degree of the fit polynomial and of the bin sizes in $x$ and $y$.

\section{Results and discussion}

Our results for $P(s)$ are presented in Fig. 22. For small values of $\mu$, we probe the regime of "weak non-hermiticity" [6] where the typical size of the real part of the eigenvalues is of the order 

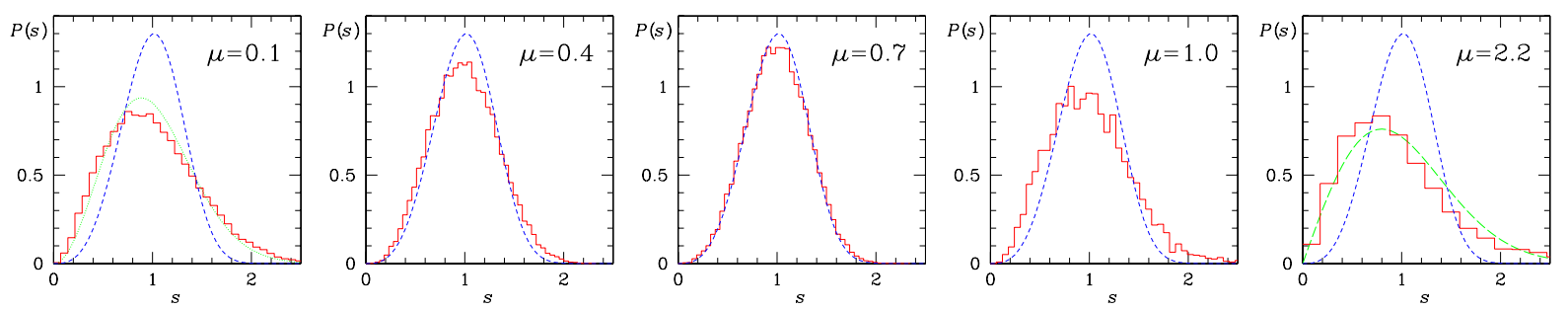

Figure 2. Nearest-neighbor spacing distribution of the Dirac operator with complex eigenvalues for various values of $\mu$. The histograms represent the lattice data. The short-dashed curve is the Ginibre distribution of Eq. (2), the dotted curve in the left plot the Wigner distribution of Eq. (11), and the long-dashed curve in the right plot the Poisson distribution of Eq. (3), respectively.

of the average separation of the imaginary parts, see Fig. 1] (our definitions differ from Ref. [6] by a factor of $i$ so that it would be more appropriate to speak of "weak non-anti-hermiticity"). Some analytical results are known [6] in this regime, but the spacing distribution is only given as an expansion of $P\left(s, z_{0}\right)$ for small $s$, where $z_{0}$ is the location in the complex plane (i.e., no unfolding is performed). Therefore, we have no closed analytical result to compare with, and presumably it would be very difficult to derive one. However, something can be said about the small-s behavior of $P(s)$. For the Ginibre ensemble, we have $P_{\mathrm{G}}(s) \propto s^{3}$ for $s \ll 1$. In the regime of weak non-hermiticity, it is known that $P(s)$ starts with a power smaller than three [6]. This is conform with our results for $\mu=0.1$ in Fig. 2 where the Wigner surmise $P_{\mathrm{W}}(s)$ of Eq. (值) for the corresponding hermitian matrix with $\mu=0$ is inserted.

Increasing $\mu$, we observe stronger level repulsion leading to quite nice agreement of the data for $\mu=0.7$ with the prediction from the Ginibre ensemble. For this value of $\mu$, we are in the regime of "strong non-hermiticity" where the Ginibre ensemble applies.

For values of $\mu$ larger than 0.7 , the lattice data deviate substantially from the Ginibre distribution. The main question is if our result for $\mu=2.2$ in Fig. 2 should be interpreted as a transition to Poisson behavior, or if the agreement of the data at this particular value of $\mu$ with $P_{\overline{\mathrm{P}}}(s)$ of Eq. (3) is purely accidental. We have to keep in mind that we neglected $\mu$ in the generation of the equilibrium gauge fields. Therefore, we are not entitled to make any definite statements on possible connections between the finite density phase transition (expected at smaller values of $\mu$ ) and the observed deviations from Ginibre behavior. Nonetheless, these deviations are interesting and deserve further study.

In conclusion, we have investigated the nearestneighbor spacing distribution of the lattice Dirac operator with finite chemical potential. The data agree with the RMT prediction for the Ginibre ensemble for $\mu=0.7$ and show deviations for smaller and larger values of $\mu$. This is somewhat different from the situation at $\mu=0$ where the data agree with the RMT results [1].

Acknowledgments. This work was supported in part by FWF project P10468-PHY and DFG grant We 655/15-1. We thank J.J.M. Verbaarschot for useful discussions.

\section{REFERENCES}

1. M.A. Halasz and J.J.M. Verbaarschot, Phys. Rev. Lett. 74 (1995) 3920; R. Pullirsch, K. Rabitsch, T. Wettig, and H. Markum, Phys. Lett. B 427 (1998) 119.

2. O. Bohigas, M.-J. Giannoni, and C. Schmit, Phys. Rev. Lett. 52 (1984) 1.

3. J. Ginibre, J. Math. Phys. 6 (1965) 440.

4. R. Grobe, F. Haake, and H.-J. Sommers, Phys. Rev. Lett. 61 (1988) 1899.

5. M.A. Stephanov, Phys. Rev. Lett. 76 (1996) 4472.

6. Y.V. Fyodorov, B.A. Khoruzhenko, and H.-J. Sommers, Phys. Rev. Lett. 79 (1997) 557. 